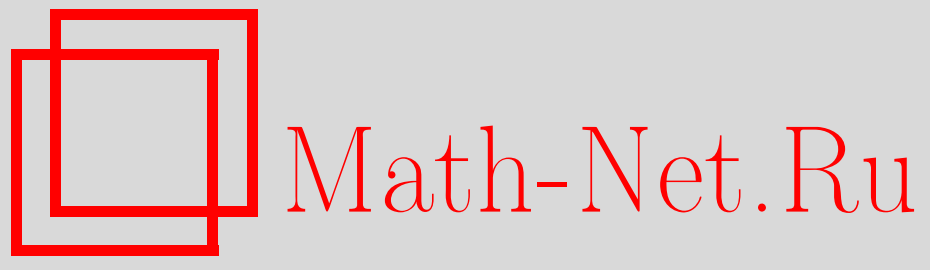

W. Bryc, W. Matysiak, Wilson's $6-j$ laws and stitched Markov processes, Теория вероятн. и ее примен., 2015, том 60, выпуск $2,333-356$

DOI: https://doi.org/10.4213/tvp4622

Использование Общероссийского математического портала Math-Net.Ru подразумевает, что вы прочитали и согласны с пользовательским соглашением http: //www . mathnet.ru/rus/agreement

Параметры загрузки:

IP : 54.210 .77 .194

26 апреля 2023 г., 18:24:14

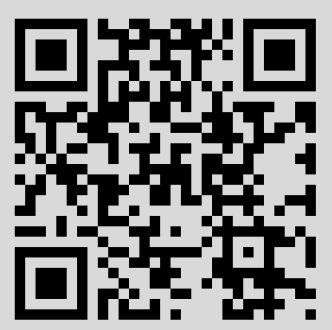




\section{WILSON'S $6-j$ LAWS AND STITCHED MARKOV PROCESSES ${ }^{1)}$}

Построены новые примеры квадратичных харнессов (quadratic harnesses) с максимальным параметром $\gamma$. Конструкция основана на квадратичных функциях цепей Маркова, которые возникают в последствие временной параметризации $6-j$ законов Вильсона. Конструкция состоит из сшивания условно независимых копий таких процессов Маркова.

Ключевые слова и фразы: марковские процессы с линейной регрессией, квадратичные условные дисперсии, ортогональные полиномы.

1. Introduction. This paper is a part of a larger research project in which we study random processes that have linear regressions and quadratic conditional variances, which we call quadratic harnesses. The distinguishing and unusual feature of this approach is the use of two-sided conditioning, so that we treat the stochastic processes as random fields with one-dimensional index set. Informally, quadratic harnesses are defined by the following properties. If the starting point $\left(t_{0}, x_{0}\right)$ and the destination point $\left(t_{1}, x_{1}\right)$ are known, then

(i) on average a quadratic harness follows the straight line that connects the end points;

(ii) the average deviation (variance) from such a straight line is a quadratic function of the end-points.

Examples of quadratic harnesses include processes which are frequently used as models of random phenomena like the Wiener, Poisson, gamma, and negative binomial processes, as well as some less common Markov processes such as the generalized Waring process [12] or the hyperbolic secant process. More exotic examples of mathematical interest arise from free Lévy processes of noncommutative probability theory, and as Markov processes constructed from the orthogonality measures of the Askey-Wilson polynomials [3].

${ }^{*}$ Department of Mathematical Sciences, University of Cincinnati, PO Box 210025, Cincinnati, USA; e-mail: wlodzimierz.bryc@uc.edu

${ }^{* *}$ Wydział Matematyki i Nauk Informacyjnych, Politechnika Warszawska, ul. Koszykowa 75, 00-662 Warsaw, Poland; e-mail: matysiak@mini.pw.edu.pl

1) This work was partially supported by NSF grant № DMS-0904720. 
When viewed as quadratic harnesses, these seemingly diverse Markov processes admit a common parametric description with five parameters $\eta, \theta \in \mathbf{R}, \sigma, \tau \geqslant 0$, and $\gamma \leqslant 1+2 \sqrt{\sigma \tau}$ (these constraint are established in [2, Theorem 2.2]) for $T=(0, \infty)$ only). However, the precise range of parameters that correspond to actual processes is not known. In particular, while the existing examples exhaust the range $-1 \leqslant \gamma \leqslant 1-2 \sqrt{\sigma \tau}$, there are only a few examples with $\gamma>1-2 \sqrt{\sigma \tau}$. In fact, [9, Section 3, Remark 2] identifies an obstruction to existence of quadratic harnesses with $\gamma>1-2 \sqrt{\sigma \tau}$. The only previously known examples with $\gamma=1+2 \sqrt{\sigma \tau}$ were constructed in [5] from pairs of Lévy processes with randomized parameter; parameters $\eta, \theta$ for such processes are tied by an additional relation $\eta \sqrt{\tau}=\theta \sqrt{\sigma}$, see $[4$, Proposition 2.10(iii)]. The construction avoids «the obstruction» as these processes do not have higher moments.

In this paper we construct additional examples of quadratic harnesses with $\gamma=1+2 \sqrt{\sigma \tau}$, with uncoupled parameters $\eta, \theta$. Szablowski's obstruction does not apply to our construction, as our processes have finite support so the higher-order orthogonal polynomials are degenerate. This raises some hope that perhaps other finitely supported processes may lead to quadratic harnesses with values $1-2 \sqrt{\sigma \tau}<\gamma<1+2 \sqrt{\sigma \tau}$.

The technical parts of the proofs in this paper are essentially computational, and not truly illuminating. However, we rely on a number of identities which we hope will later serve as a guide towards the more difficult constructions based on discrete orthogonality for Askey-Wilson and $q$-Racah polynomials. We expect that such constructions will be «universal», with all known cases arising as appropriate limits.

The paper is organized as follows. The main result, Theorem 1.1, is stated together with some introductory material on quadratic harnesses in Section 1.1. Basically, the rest of the paper is devoted to proving the main result. We outline the main steps of the proof in Section 1.2. In Section 2 we use Wilson $6-j$ laws to construct quadratic harnesses on $(0,1)$ and on $(1, \infty)$ - see Propositions 2.3 and 2.5. In order to extend the process to $(0, \infty)$, in Section 3 we represent two Markov chains from Section 3 as a mixture of «simpler» Markov processes. We also confirm that each of these Markov chains transforms into a quadratic harness with $\gamma=1$ and $\sigma=0$ (which is our justification for the adjective «simpler» in the previous sentence.) In Section 3.3 we stitch together a pair of such quadratic harnesses into the quadratic harness on $(0, \infty)$, thus extending the process from Proposition 2.3 to the maximal time domain. Appendix 3.5. contains computational results on Wilson's laws.

1.1. Quadratic harnesses. In [2] the authors consider squareintegrable stochastic processes on $(0, \infty)$ such that for all $t, s>0$,

$$
\mathbf{E}\left(Z_{t}\right)=0, \quad \mathbf{E}\left(Z_{s} Z_{t}\right)=\min \{s, t\},
$$


and for $s<t<u, \mathbf{E}\left(Z_{t} \mid \mathscr{F}_{s, u}\right)$ is a linear function of $Z_{s}, Z_{u}$, and $\operatorname{Var}\left[Z_{t} \mid \mathscr{F}_{s, u}\right]$ is a quadratic function of $Z_{s}, Z_{u}$ (it is assumed that the functions have coefficients which are some nonrandom functions of $s, t, u)$. Here, $\mathscr{F}_{s, u}$ is the two-sided $\sigma$-field generated by $\left\{Z_{r}: r \in(0, s] \cup[u, \infty)\right\}$. Then $(1.1)$ implies that

$$
\mathbf{E}\left(Z_{t} \mid \mathscr{F}_{s, u}\right)=\frac{u-t}{u-s} Z_{s}+\frac{t-s}{u-s} Z_{u}
$$

for all $s<t<u$, which is sometimes referred to as a harness condition, see, e.g., [8].

While there are numerous examples of harnesses, the assumption of quadratic conditional variance is more restrictive. For example, all integrable Lévy processes are harnesses, but as determined by Wesołowski [10], only a few of them are also quadratic harnesses. Under certain technical assumptions, [2, Theorem 2.2] asserts that quadratic variance has the following form: there exist numerical constants $\eta, \theta \in \mathbf{R}, \sigma, \tau \geqslant 0$, and $\gamma \leqslant 1+2 \sqrt{\sigma \tau}$ such that for all $s<t<u$,

$$
\begin{aligned}
\operatorname{Var}\left[Z_{t} \mid \mathscr{F}_{s, u}\right]= & \frac{(u-t)(t-s)}{u(1+\sigma s)+\tau-\gamma s} \\
& \quad \times\left(1+\eta \frac{u Z_{s}-s Z_{u}}{u-s}+\theta \frac{Z_{u}-Z_{s}}{u-s}+\sigma \frac{\left(u Z_{s}-s Z_{u}\right)^{2}}{(u-s)^{2}}\right. \\
& \left.\quad+\tau \frac{\left(Z_{u}-Z_{s}\right)^{2}}{(u-s)^{2}}-(1-\gamma) \frac{\left(Z_{u}-Z_{s}\right)\left(u Z_{s}-s Z_{u}\right)}{(u-s)^{2}}\right) .
\end{aligned}
$$

D e f i n it i o n 1.1. Let $T$ be an open interval $T$ which may be all of or a proper subset of $(0, \infty)$. We will say that a square-integrable stochastic process $\left(Z_{t}\right)_{t \in T}$ is a quadratic harness on $T$ with parameters $(\eta, \theta, \sigma, \tau, \gamma)$ if it satisfies the following.

(i) We assume that the first two moments are given by (1.1).

(ii) We assume (1.2) and that the one-sided conditional moments are as follows: for $0<s \leqslant t \leqslant u$ in $T$,

$$
\begin{aligned}
& \mathbf{E}\left[Z_{t} \mid \mathscr{F}_{\geqslant u}\right]=\frac{t}{u} Z_{u}, \\
& \mathbf{E}\left[Z_{t} \mid \mathscr{F}_{\leqslant s}\right]=Z_{s},
\end{aligned}
$$

(iii) We assume that (1.3) holds on $T$. We also assume that the one-sided conditional variances are given by

$$
\begin{aligned}
\operatorname{Var}\left[Z_{t}, \mid \mathscr{F}_{\leqslant s}\right] & =\frac{t-s}{1+\sigma s}\left(\sigma Z_{s}^{2}+\eta Z_{s}+1\right), \\
\operatorname{Var}\left[Z_{t} \mid \mathscr{F}_{\geqslant u}\right] & =\frac{t(u-t)}{u+\tau}\left(\tau \frac{Z_{u}^{2}}{u^{2}}+\theta \frac{Z_{u}}{u}+1\right) .
\end{aligned}
$$


We remark that on the infinite interval $T=(0, \infty)$, formulas $(1.4)-(1.7)$ follow from the other assumptions, see [2, (2.7), (2.8), (2.27), and (2.28)]. We do not know if (1.4) or (1.5) can be deduced from (1.2) on finite intervals but we suspect that this cannot be done.

We can now state our main result. Fix $N \in \mathbf{N}, A>-1 / 2, B \in$ $(-A, A+1)$, and

$$
C<-A-N+1 .
$$

Theorem 1.1. There exist a Markov quadratic harness $\left(Z_{t}\right)_{t \in(0, \infty)}$ with covariance (1.1) and conditional variance (1.3) with parameters

$$
\begin{aligned}
\eta & =-\frac{A(B+C+N)+C(B+C)+N(N-B)}{\sqrt{N(A+C)(B+C)(A-B+N)(N-1-B-C)}}, \\
\theta & =-\frac{A(B+C+N)-B(B+C-N)+2 C N}{\sqrt{N(A+C)(B+C)(A-B+N)(N-1-B-C)}}, \\
\sigma & =\tau=\frac{1}{N-1-B-C} \\
\gamma & =1+2 \sqrt{\sigma \tau}=\frac{B+C-N-1}{B+C-N+1}
\end{aligned}
$$

1.2. Outline of proof. The proof of Theorem 1.1 is long and contains computational components that might be difficult to follow. We therefore begin with an informal description of our construction.

The basic plan of the construction is modeled in [5], and consists of stitching a pair of conditionally independent Markov processes into one process. The basic plan in [5] was to choose a pair of Lévy processes with common parameter $\theta$ and then «randomize» $\theta$ by a well chosen law. Here we proceed similarly. We choose random variable $\Theta$, and a pair of Markov processes $\left(Y_{t}^{(\Theta)}\right)_{t \in \mathscr{T}}$ and $\left(\widetilde{Y}_{t}^{(\Theta)}\right)_{t \in \widetilde{T}}$ with quadratic conditional variances and linear regressions. A pair of Möbius transformations puts these two processes together on adjacent intervals as $\left(Z_{t}\right)_{t \in(0,1)}$ and $\left(Z_{t}\right)_{t \in(1, \infty)}$. We then use Lemma 3.1 to show that together with $Z_{1}$ (which is just the standardized $\Theta)$, these processes define a quadratic harness on $(0, \infty)$.

There are two fairly computational tools that we use. One is Proposition B.1 from [4] which is an elementary but tedious recalculation of the conditional moments under a Möbius transformation of time. The second is Lemma 3.1 from [5] which gives conditions under which the three components $\left(Z_{t}\right)_{t<1}, Z_{1}$, and $\left(Z_{t}\right)_{t>1}$ form together a quadratic harness. Verification of assumptions of Lemma 3.1 is rather tedious: we first consider separately two unconditional processes $\left(Y_{t}\right)=\left(Y_{t}^{(\Theta)}\right)_{t \in \mathscr{T}}$ and $\left(Y_{t}^{\prime}\right)=\left(\widetilde{Y}_{t}^{(\Theta)}\right)_{t \in \widetilde{\mathscr{T}}}$ and derive formulas for the mean, covariance, and the fist two conditional moments. Then in Section 3.3 we consider the two processes together and verify conditions on the conditional moments of $Z_{1}$. 
2. Two quadratic harnesses. In this section we construct two quadratic harnesses $\left(Z_{t}\right)_{t \in(0,1)}$ and $\left(Z_{t}\right)_{t \in(1, \infty)}$ that form «two parts» of the quadratic harness to be later stitched into one quadratic harness in Theorem 1.1. At this time, the processes are defined separately. The construction consists of several steps:

(i) We first introduce a pair of Markov chains $\left(\zeta_{t}\right)$ and $\left(\zeta_{t}^{\prime}\right)$ on the finite state space $\{0,1, \ldots, N\}$ with transition probabilities based on Wilson's distributions. The computational components here is verification of ChapmanKolmogorov equations for the nonhomogeneous transition matrices.

(ii) We then apply a time-dependent quadratic transformation to define a pair of Markov processes $\left(Y_{t}\right)$ and $\left(Y_{t}^{\prime}\right)$. In order to be able to transform $\left(Y_{t}\right)$ and $\left(Y_{t}^{\prime}\right)$ into quadratic harnesses $\left(Z_{t}\right)_{t \in(0,1)}$ and $\left(Z_{t}\right)_{t \in(1, \infty)}$, we need to compute the mean, the covariance, and the first two conditional moments. Calculations are involved but straightforward, as we can rely on algebraic identities for Wilson's distributions to compute the conditional laws.

(iii) The final transformation of $\left(Y_{t}\right)$ and $\left(Y_{t}^{\prime}\right)$ into quadratic harnesses $\left(Z_{t}\right)_{t \in(0,1)}$ and $\left(Z_{t}\right)_{t \in(1, \infty)}$ is based on Möbius transformation of the time intervals from Proposition B.1.

2.1. Wilson's distributions. Our basic tool is a family of finitelysupported Wilson's distributions that arose in his study of a family of orthogonal polynomials. As in $[11,(3.4)]$ we fix integer $N \geqslant 1$ and real parameters $a, b, c$. For $k=0,1, \ldots, N$, we consider

$$
p_{k, N}(a, b, c)=C_{N}(a, b, c) \frac{(2 a)_{k}(a+1)_{k}(a+b)_{k}(a+c)_{k}(-N)_{k}}{k !(a)_{k}(a-b+1)_{k}(a-c+1)_{k}(2 a+N+1)_{k}},
$$

where the normalizing constant is

$$
C_{N}(a, b, c)=\frac{(a-b+1)_{N}(a-c+1)_{N}}{(2 a+1)_{N}(1-b-c)_{N}}
$$

and $(a)_{k}=\Gamma(a+k) / \Gamma(a)=a(a+1) \cdots(a+k-1)$ is the Pochhammer symbol. The probability measure given by (2.1) is also the orthogonality measure of Racah polynomials, see, e.g., [7].

It will be convenient to include in (2.1) also the degenerate case $N=0$; to do so we define $p_{0,0}(a, b, c)=1$.

Expression (2.1) defines positive numbers under various constraints on the parameters $a, b, c$. A version of a sufficient condition $[11,(3.5)]$ in our notation is

$$
a>-1 / 2, \quad b \in(-a, a+1), \quad \text { and } \quad c<-a-N+1 .
$$

Noting that (2.1) is symmetric in $b, c$, Wilson's remark about swapping his first two parameters can be restated as another sufficient condition for positivity:

$$
a<-1 / 2, \quad b>-a, \quad \text { and } \quad a+N<c<1-a-N .
$$


From $[11,(3.4)]$ applied to $m=n=0$ one reads out that

$$
\sum_{k=0}^{N} p_{k, N}(a, b, c)=1
$$

so when positivity conditions hold, formula (2.1) defines a four-parameter family of probability measures on $\{0,1, \ldots, N\}$. Under suitable choices of the parameters, these measures will be used as the transition probabilities for the Markov chains that we consider in what follows.

Wilson's weights satisfy an algebraic relation in Lemma A.1 which we will use to verify Markov property and compute conditional laws. Lemma A.2 gives the first two moments of a quadratic function that we use to define the processes.

2.2. Markov chains. In this section we construct auxiliary Markov chains $\left(\zeta_{t}\right)$ and $\left(\zeta_{t}^{\prime}\right)$ on the finite state space $\{0,1, \ldots, N\}$.

For $s<t$, define the matrix $P_{s, t}=\left[p_{s, t}(k, n)\right]_{0 \leqslant k, n \leqslant N}$ with entries

$$
p_{s, t}(k, n)=p_{n-k, N-k}\left(A+\frac{t}{2}+k,-A-s+\frac{t}{2}-k, C-\frac{t}{2}\right)
$$

if $0 \leqslant k \leqslant n \leqslant N$; we take $p_{s, t}(k, n)=0$ for all other values $k, n \in\{0, \ldots, N\}$.

It turns out that (2.6) are probabilities on two disjoint time intervals.

Lemma 2.1. Numbers (2.6) are nonnegative for $-(A+B)<s<t$ and for $s<t<C-A-N$.

$\mathrm{P}$ r o o f. We remark that the two time intervals are disjoint: $C-A-$ $N<-(A+B)$ as $C-A-N<-2 A-2 N+1<-2 N \leqslant-2$ while $A+B>0$.

Suppose $-(A+B)<s<t$. To verify that for $0 \leqslant k \leqslant n \leqslant N$ we have $p_{s, t}(k, n) \geqslant 0$ we verify that assumption (2.3) with $N$ replaced by $N-k$ holds for $a=A+k+t / 2, b=-A-k-s+t / 2, c=C-t / 2$.

To check that (2.1) is positive for $s<t<C-A-N$ we use (2.4) with $N$ replaced by $N-k$, where $k<N$. Lemma 2.1 is proved.

The following shows that matrices $P_{s, t}$ satisfy Chapman-Kolmogorov equations, so when they are nonnegative, they define transition probabilities for a Markov chain.

Proposition 2.1 (Chapman-Kolmogorov equations). For $s<t<u$ and $j \leqslant n \leqslant N$,

$$
p_{s, u}(j, n)=\sum_{k=j}^{n} p_{s, t}(j, k) p_{t, u}(k, n)
$$

Furthermore,

$$
\sum_{k=j}^{N} p_{s, t}(j, k)=1 \text {. }
$$


P r o o f. To verify (2.7), we apply (A.2) with parameters $a=A+j+$ $t / 2, b=-A-j-s+t / 2, c=C-u / 2, \delta=(u-t) / 2$. Formula (2.8) is the algebraic identity (2.5) for Wilson's weights. The proposition is proved.

We now define two Markov chains with time indexes $\mathscr{T}=(-A-B, \infty)$ and $\widetilde{\mathscr{T}}=(A+N-C, \infty)$.

Let $\left(\zeta_{t}\right)_{t \in \mathscr{T}}$ be the Markov chain, starting with $\zeta_{-A-B}=0$ and with transition probabilities $\mathbf{P}\left(\zeta_{t}=n \mid \zeta_{s}=k\right)=p_{s, t}(k, n), 0 \leqslant k \leqslant n \leqslant N$. In particular, the univariate laws of the Markov chain are

$$
\mathbf{P}\left(\zeta_{t}=j\right)=p_{-A-B, t}(0, j)=p_{j, N}\left(A+\frac{t}{2}, B+\frac{t}{2}, C-\frac{t}{2}\right) .
$$

The second Markov chain can informally be described as the time inversion $\zeta_{t}^{\prime}=\zeta_{-t}$ of «the second branch» of $\zeta_{t}$ for $t<C-A-N$. Recall that Lemma 2.1 says that the transition probabilities (2.6) are well defined also for $s<t<C-A-N$. We will use this interpretation to derive formulas for the moments. Formally, let $\left(\zeta_{t}^{\prime}\right)_{t \in \widetilde{T}}$ be the Markov chain, starting with $\zeta_{A+N-C}^{\prime}=N$ and with transition probabilities

$\check{p}_{s, t}(k, n)=p_{n, k}\left(A-\frac{t}{2}, B-\frac{t}{2}, A-s+k+\frac{t}{2}\right), \quad k=0, \ldots, N, \quad n=0, \ldots, k$.

(The transition probabilities are recalculated from the transition probabilities for $\zeta_{t}$.) In particular, the univariate laws are

$$
\mathbf{P}\left(\zeta_{t}^{\prime}=k\right)=\check{p}_{t}(k):=p_{k, N}\left(A-\frac{t}{2}, B-\frac{t}{2}, C+\frac{t}{2}\right), \quad k=0, \ldots, N,
$$

2.3. Two Markov processes with quadratic conditional variances. We now transform Markov chains from Section 2.2 into Markov processes with linear regressions and quadratic conditional variances.

For $t \in \mathscr{T}$, consider the process

$$
Y_{t}=\left(2 A+t+\zeta_{t}\right) \zeta_{t}+A(A+t)=\left(A+t+\zeta_{t}\right)\left(A+\zeta_{t}\right) .
$$

Lemma 2.2. $\left(Y_{t}\right)$ is a Markov process.

P r o o f. Markov property follows from the fact that the lines $\ell_{k}(t)=$ $(A+t+k)(A+k)$ are distinct for $k=0,1, \ldots, N$ and do not intersect over $t \in \mathscr{T}$. Indeed, $\ell_{k}$ and $\ell_{j}$ intersect at $t=-(2 A+j+k)<-2 A-1<$ $-(A+B)$. Therefore, $(2.12)$ is a one-to-one transformation, which implies Markov property. Lemma 2.2 is proved.

We will want to use Proposition B.1 to transform $\left(Y_{t}\right)$ into the quadratic harness. To do this, we need to know that $\mathbf{E}\left(Y_{t}\right)$ is a linear function of $t$, that for $s<t$ the covariance $\operatorname{Cov}\left(Y_{s}, Y_{t}\right)$ is the product of linear functions of $s$ and $t$, that (1.2) holds, and that the two-sided conditional variances are quadratic. 


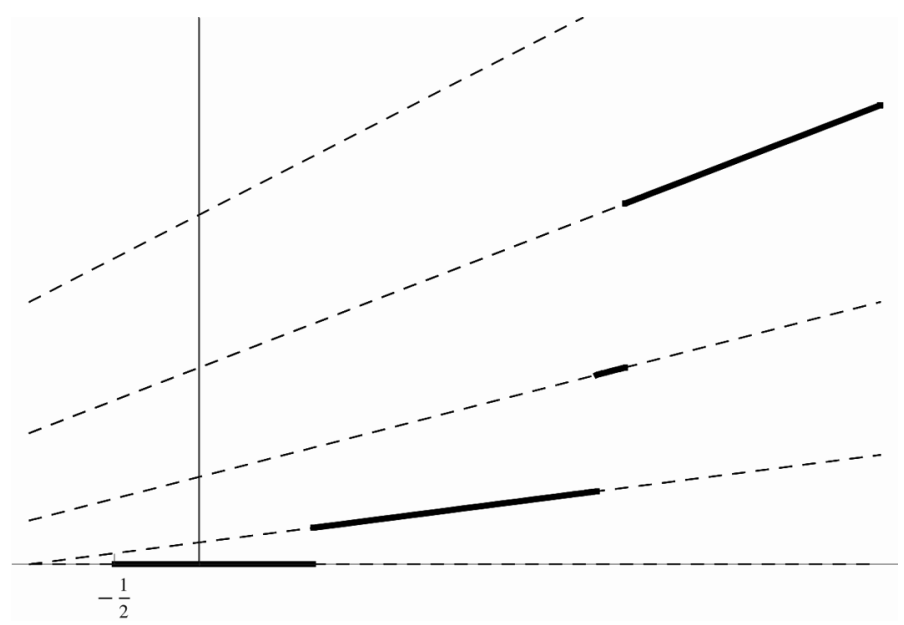

Fig. 1. Trajectories of process $\left(Y_{t}\right)_{t \in \mathscr{T}}$ lie on the family of lines. The process starts at line $\ell_{0}=(A+t) A$, at $t=-A-B$ and jumps up until it stops on a random line $\ell_{\Theta}=(A+t+\Theta)(A+\Theta)$ that it follows to $\infty$. (Here, $A=0, B=1 / 2, N=4, \Theta=3$; for more on $\Theta$, see (3.10).)

Proposition 2.2. For $0<s<t<u$ we have

$$
\begin{gathered}
\mathbf{E}\left(Y_{t}\right)=\frac{A(B+C)(A+N)+N B C}{B+C-N}+t \frac{A(B+C)+N C}{B+C-N} \\
\operatorname{Cov}\left(Y_{s}, Y_{t}\right)=\frac{A-C+N+t}{A-C+N+s} \operatorname{Var}\left(Y_{s}\right) \\
=-\frac{N(A+C)(B+C)(A-B+N)(A+B+s)(A-C+N+t)}{(B+C-N)^{2}(B+C-N+1)} \\
\mathbf{E}\left(Y_{t} \mid Y_{s}, Y_{u}\right)=\frac{(u-t) Y_{s}+(t-s) Y_{u}}{u-s}, \\
\operatorname{Var}\left(Y_{t} \mid Y_{s}, Y_{u}\right)=\frac{(u-t)(t-s)}{u-s+1}\left(\frac{\left(Y_{u}-Y_{s}\right)^{2}}{(u-s)^{2}}-\frac{u Y_{s}-s Y_{u}}{u-s}\right)
\end{gathered}
$$

The proof is computational. We begin by computing the mean and the variance of $Y_{t}$.

Lemma 2.3. $\left(Y_{t}\right)_{t \in \mathscr{T}}$ has the mean (2.13) and the variance

$$
\operatorname{Var}\left(Y_{t}\right)=-\frac{N(A+C)(B+C)(A-B+N)}{(B+C-N)^{2}(B+C-N+1)}(A+B+t)(A-C+N+t) \text {. }
$$

$\mathrm{P}$ r o o $\mathrm{f}$. The formulas for the mean and the variance are now recalculated from Lemma A.2, noting that $Y_{t}$ is equidistributed with $Y+A(A+t)$, where $Y$ is given by (A.6). (The parameters are listed in (2.9).) Lemma 2.3 is proved.

Further, we verify that Markov process $\left(Y_{t}\right)_{t}$ has linear regressions and quadratic conditional variances under one-sided conditioning. 
Lemma 2.4. For $s<t$ we have

$$
\begin{aligned}
\mathbf{E}\left(Y_{t} \mid Y_{s}\right) & =Y_{s} \frac{A-C+N+t}{A-C+N+s}-\frac{C(A+N)(t-s)}{A-C+N+s}, \\
\operatorname{Var}\left(Y_{t} \mid Y_{s}\right)= & (A-C+N+t)(t-s) \\
& \times \frac{\left(Y_{s}+C(s-C)\right)\left(Y_{s}-(A+N)(s+A+N)\right)}{(A-C+N+s)^{2}(A-C+N+s-1)} .
\end{aligned}
$$

P r o o f. These formulas follow from Lemma A.2, as the conditional law of $Y_{t}-A(A+t)$ given $\zeta_{s}$ is the same as the law of (A.6) that is induced by the transition probabilities $p_{s, t}(k, n)=p_{n-\xi_{s}, N-\xi_{s}}\left(A+t / 2+\xi_{s},-A-s+\right.$ $\left.t / 2-\xi_{s}, C-t / 2\right)$. Lemma 2.4 is proved.

$\mathrm{P}$ r o of of $\mathrm{P}$ r o p o s i t i o $\mathrm{n}$ 2.2. To compute the covariance we recall that

$$
\operatorname{Cov}(X, Y)=\mathbf{E}(\operatorname{Cov}(X, Y \mid U))+\operatorname{Cov}(\mathbf{E}(X \mid U), \mathbf{E}(Y \mid U)) .
$$

Using (2.20) with $X=U=Y_{s}, Y=Y_{t}$, from (2.17) and (2.18) we get

$$
\begin{aligned}
& \operatorname{Cov}\left(Y_{s}, Y_{t}\right)=\frac{A-C+N+t}{A-C+N+s} \operatorname{Var}\left(Y_{s}\right) \\
& =-\frac{N(A+C)(B+C)(A-B+N)(A+B+s)(A-C+N+t)}{(B+C-N)^{2}(B+C-N+1)} .
\end{aligned}
$$

We now compute the two-sided conditional moments. The two-sided conditional distribution is

$$
\begin{aligned}
\mathbf{P}\left(\xi_{t}=j \mid \xi_{s}=k, \xi_{u}=m\right) & =\frac{\mathbf{P}\left(\xi_{t}=j \mid \xi_{s}=k\right) \mathbf{P}\left(\xi_{u}=m \mid \xi_{t}=j\right)}{\mathbf{P}\left(\xi_{u}=m \mid \xi_{s}=k\right)} \\
& =\frac{p_{s, t}(k, j) p_{t, u}(j, m)}{p_{s, u}(k, m)}
\end{aligned}
$$

The conditional probability is well defined and nonzero only for $k \leqslant j \leqslant m \leqslant$ $N$, and then we have

$$
\mathbf{P}\left(\xi_{t}=j \mid \xi_{s}, \xi_{u}\right)=p_{j-\xi_{s}, \xi_{u}-\xi_{s}}\left(A+\xi_{s}+\frac{t}{2},-s+\frac{t}{2}-A-\xi_{s}, \xi_{u}+A-\frac{t}{2}+u\right) .
$$

Indeed, from (A.1) it follows that

$$
\begin{gathered}
\frac{p_{j-k, N-k}(a, b, c+\delta) p_{m-j, N-j}(a+j-k+\delta,-a-j+k+\delta, c)}{p_{m-k, N-k}(a+\delta, b+\delta, c)} \\
=p_{j-k, m-k}(a, b, a+m-k+2 \delta) .
\end{gathered}
$$

Taking $a=A+t / 2+k, b=-A-s+t / 2-k, c=C-u / 2$ and $\delta=(u-t) / 2$, we get (2.21). 
We now use (2.21) to compute the two-sided conditional moments. For fixed $\xi_{s}=k, \xi_{u}=m$, we use (A.7) with $a=A+k+t / 2, b=-s+t / 2-A-k$, $c=m+A-t / 2+u$ and $N=m-k$ to compute $\mathbf{E}\left(Y \mid \xi_{s}, \xi_{u}\right)$, where $Y=\left(\xi_{t}-k\right)\left(2 a+\xi_{t}-k\right)$. Since $\mathbf{P}\left(Y_{t}=y \mid \xi_{s}=k, \xi_{u}=m\right)=\mathbf{P}(Y+(A+$ $k)(A+k+t)=y)$, reverting to $k=\xi_{s}$ and $m=\xi_{u}$, we obtain

$$
\begin{aligned}
\mathbf{E}\left(Y_{t} \mid \xi_{s}, \xi_{u}\right) & =\left(A+\xi_{s}\right)\left(A+t+\xi_{s}\right)+\frac{(t-s)\left(\xi_{u}-\xi_{s}\right)\left(2 A+u+\xi_{s}+\xi_{u}\right)}{u-s} \\
& =\frac{u-t}{u-s} \xi_{s}\left(2 A+s+\xi_{s}\right)+\frac{t-s}{u-s} \xi_{u}\left(2 A+u+\xi_{u}\right)+A(A+t) .
\end{aligned}
$$

Hence a calculation based on (2.12) gives (2.15).

Further, we use (A.8) with $N=\xi_{u}-\xi_{s}, a=A+\xi_{s}+t / 2, b=-s+$ $t / 2-A-\xi_{s}$, and $c=\xi_{u}+A-t / 2+u$ to compute the conditional variance:

$$
\begin{aligned}
& \operatorname{Var}\left(Y_{t} \mid \xi_{s}, \xi_{u}\right) \\
& \quad=C_{t, s, u}\left(\xi_{u}-\xi_{s}\right)\left(\xi_{u}-\xi_{s}+u-s\right)\left(2 A+s+\xi_{s}+\xi_{u}\right)\left(2 A+u+\xi_{s}+\xi_{u}\right),
\end{aligned}
$$

where

$$
C_{t, s, u}=\frac{(t-s)(u-t)}{(u-s+1)(u-s)^{2}} .
$$

This gives (2.16). Proposition 2.2 is proved.

Proposition 2.3. $\left(Y_{t}\right)_{t \in \mathscr{T}}$ can be transformed into a quadratic harness $\left(Z_{t}\right)_{t}$ on $\mathscr{T}^{\prime}=(0,1)$ with covariance $(1.1)$ and the conditional variance $(1.3)$ with parameters $(1.9)-(1.12)$.

Furthermore, the one-sided conditional moments of $\left(Z_{t}\right)$ are given by (1.4)-(1.7)

P r o o f. We shall use Proposition B.1. We set

$$
M=(N-B-C)^{-1} \sqrt{\frac{N(A+C)(B+C)(A-B+N)}{(N-1-B-C)}},
$$

noting that our constraints on parameters $A, B, C, N$ imply that the expression under the radical is positive. Let $\psi=A+B, \delta=A-C+N$, so that $\delta-\psi=N-B-C>0$. We take

$$
\alpha=\frac{A(B+C)(A+N)+N B C}{B+C-N}, \quad \beta=\frac{A(B+C)+N C}{B+C-N},
$$

$\theta_{0}=0, \eta_{0}=-1$, and define

$$
X_{t}=Y_{t}-\mathbf{E} Y_{t}, \quad Z_{t}=m(t) X_{\ell(t) / m(t)},
$$

with

$$
\ell(t)=\frac{t(A-C+N)-(A+B)}{M(N-B-C)} \quad \text { and } \quad m(t)=\frac{1-t}{M(N-B-C)}
$$


as defined in Proposition B.1. Then by Proposition B.1, $\left(Z_{t}\right)$ is a quadratic harness, and the formulas follow by calculation. First,

$$
\chi=\beta^{2}-\alpha=\frac{(A+C)(B+C) N(A-B+N)}{(B+C-N)^{2}}>0,
$$

SO

$$
\frac{M}{\chi}=-\frac{B+C-N}{((A+C)(B+C) N(A-B+N)(N-1-B-C))^{1 / 2}},
$$

and $\sigma=\tau=1 /(N-1-B-C)$. Then,

$$
\eta=\frac{M(2 \beta-\delta)}{\chi}=\frac{M}{\chi} \frac{N^{2}+(A-B) N+(A+C)(B+C)}{B+C-N}
$$

and

$$
\theta=\frac{M(2 \beta-\psi)}{\chi}=\frac{M}{\chi} \frac{(A-B)(B+C)+(A+B+2 C) N}{B+C-N} .
$$

To complete the proof, we verify that the one-sided conditional moments are as stated. From (2.18) we get

$$
\mathbf{E}\left(X_{t} \mid X_{s}\right)=\frac{A-C+N+t}{A-C+N+s} X_{s},
$$

therefore (note that $t \mapsto \ell(t) / m(t)$ is increasing)

$$
\mathbf{E}\left(X_{\ell(t) / m(t)} \mid X_{\ell(s) / m(s)}\right)=\frac{1-s}{1-t} X_{\ell(s) / m(s)} .
$$

Hence $\mathbf{E}\left(Z_{t} \mid Z_{s}\right)=Z_{s}$.

Further, from (2.19) we get

$$
\begin{aligned}
& \operatorname{Var}\left(X_{t} \mid X_{s}\right)=\operatorname{Var}\left(Y_{t} \mid Y_{s}\right)=\frac{(A-C+N+t)(t-s)}{(A-C+N+s)^{2}(A-C+N+s-1)} \\
& \quad \times\left[X_{s}+C(s-C)+\mathbf{E} Y_{s}\right]\left[X_{s}-(A+N)(s+A+N)+\mathbf{E} Y_{s}\right]
\end{aligned}
$$

and

$$
\begin{aligned}
& \operatorname{Var}\left(X_{\ell(t) / m(t)} \mid X_{\ell(s) / m(s)}\right) \frac{(1-s)^{2}(s-t)}{(1-t)^{2}(1+B+C-N-s)} \\
& \quad \times\left[X_{\ell(s) / m(s)}+\frac{(A+C)(B+C)}{s-1}\right]\left[X_{\ell(s) / m(s)}+\frac{N(A-B+N)}{s-1}\right] .
\end{aligned}
$$

This gives

$$
\operatorname{Var}\left(Z_{t} \mid Z_{s}\right)=m^{2}(t) \operatorname{Var}\left(X_{\ell(t) / m(t)} \mid X_{\ell(s) / m(s)}\right)=\frac{t-s}{1+\sigma s}\left(\sigma Z_{s}+\eta Z_{s}+1\right) .
$$

For a quick proof of (1.4) and (1.7) one can consider the time-reversal $\check{X}_{t}=Y_{-t}, t \in \check{\mathscr{T}}:=-\mathscr{T}$. The details are omitted. Proposition 2.3 is proved. 
2.3.1. The second Markov process. The second Markov process that we wish to consider is

$$
Y_{t}^{\prime}=\left(t-A-\zeta_{t}^{\prime}\right)\left(A+\zeta_{t}^{\prime}\right),
$$

where $\left(\zeta_{t}^{\prime}\right)_{t \in \widetilde{T}}$ is the Markov chain from Section 2.2.

Since $\left(\widetilde{\zeta}_{t}^{\prime}\right)_{t \in \widetilde{T}}$ has the same transition probabilities as the time reversal of the original process $\left(\zeta_{-t}\right)_{t \in-} \widetilde{\mathscr{T}}$, we see that properties of $\left(Y_{t}^{\prime}\right)$ can be read out from the relation $Y_{t}^{\prime}=-Y_{-t}$. We deduce the following version of Proposition 2.2.

Proposition 2.4. We have

$$
\mathbf{E}\left(Y_{t}^{\prime}\right)=t \frac{A(B+C)+N C}{B+C-N}-\frac{A(B+C)(A+N)+N B C}{B+C-N} .
$$

For $s<t$ we have

$$
\begin{aligned}
& \operatorname{Cov}\left(Y_{s}^{\prime}, Y_{t}^{\prime}\right) \\
& =-\frac{N(A+C)(B+C)(A-B+N)(t-(A+B))(s-(A-C+N))}{(B+C-N)^{2}(B+C-N+1)} .
\end{aligned}
$$

For $s<t<u$, (1.2) holds for $\left(Y_{t}^{\prime}\right)$, and

$$
\operatorname{Var}\left(Y_{t}^{\prime} \mid Y_{s}^{\prime}, Y_{u}^{\prime}\right)=\frac{(u-t)(t-s)}{u-s+1}\left(\frac{\left.Y_{u}^{\prime}-Y_{s}^{\prime}\right)^{2}}{(u-s)^{2}}+\frac{u Y_{s}^{\prime}-s Y_{u}^{\prime}}{u-s}\right) .
$$

$\mathrm{P}$ r o o f. Formulas for the mean and the variance are recalculated from see (2.13) and (2.14). Formula (2.28) is a rewrite of (2.16). Proposition 2.4 is proved.

We also have the following version of Proposition 2.3.

Proposition 2.5. $\left(Y_{t}^{\prime}\right)_{t \in \widetilde{T}}$ can be transformed into a quadratic harness $\left(Z_{t}\right)_{t}$ with $t \in(1, \infty)$, with covariance $(1.1)$ and the conditional variance $(1.3)$ with parameters (1.9)-(1.12).

Furthermore, the one-sided conditional moments of $\left(Z_{t}\right)$ are given by $(1.4)-(1.7)$.

P r o o f. Let $M$ be given by (2.22). With $X_{t}^{\prime}=Y_{t}^{\prime}-\mathbf{E}\left(Y_{t}^{\prime}\right)$ taking

$$
\ell^{\prime}(t)=\frac{t(A+N-C)-A-B}{M(N-B-C)} \quad \text { and } \quad m^{\prime}(t)=\frac{t-1}{M(N-B-C)}
$$

for $t>1$, we see that

$$
Z_{t}:=m^{\prime}(t) X_{m^{\prime}(t) / \ell^{\prime}(t)}^{\prime}
$$

defines a Markov process on $(1, \infty)$ such that $(1.1)$ holds. A longer calculation verifies (1.3); this follows from (2.28), taking into account (2.26). 
(We remark that some care is needed if one wants to use Proposition B.1 here: Proposition B.1 as stated holds for increasing Möbius transformations only, so if applied directly it gives a quadratic harness on $(0,1)$ with parameters $\eta, \theta$ swapped, i.e., $\eta$ is given by (1.10) and $\theta$ is given by (1.9). Then one needs to use time inversion $t Z_{1 / t}$ which swaps back the parameters $\eta, \theta$ and maps the process onto $(1, \infty)$. The final transformation is the same as the direct application of (2.29).)

We omit the verification of one-sided conditional moments, which are of course closely related to one-sided conditional moments of $\left(Y_{t}\right)$. Proposition 2.5 is proved.

3. Stitching together two quadratic harnesses. In this section we stitch together two quadratic harnesses from Proposition 2.3 and Proposition 2.5 by representing both processes on a single probability space as $\Theta$-conditionally independent $\Theta$-conditional Markov processes for an appropriate random variable $\Theta$.

The heuristic for this representation is fairly simple: both processes arise from the pair of Markov chains $\zeta_{t}$ and $\zeta_{t}^{\prime}=\zeta_{-t}$. In our previous construction we treated the two Markov chains separately, but it is also natural to consider $\left(\zeta_{t}\right)$ as a single Markov chain on $(-A-B, \infty] \cup(-\infty, C-A-N)$. This Markov chain starts at 0 at $t=(-A-B)$, jumps only upwards and continues to infinity with random $\Theta:=\zeta_{\infty}$ and then continues from value $\Theta$ at $t=-\infty$ until it reaches state $N$ at $t=C-A-N$.

The formal construction will consist of choosing two families of Markov chains $\left(\xi_{t}^{(K)}\right)_{t \in \mathscr{T}},\left(\widetilde{\xi}_{t}^{(K)}\right)_{t \in \widetilde{T}}$ indexed by an integer $K=0,1 \ldots$ and an appropriate random variable $\Theta$ with values $0,1, \ldots, N$ such that $\left(\xi_{t}^{(\Theta)}\right)_{t \in \mathscr{T}}$ has the same distribution as $\left(\zeta_{t}\right)_{t \in \mathscr{T}}$ and $\left(\widetilde{\xi}_{t}^{(\Theta)}\right)_{t \in \widetilde{\mathscr{T}}}$ has the same distribution as $\left(\zeta_{t}^{\prime}\right)_{t \in \widetilde{T}} \cdot$

3.1. Conditional representation. For $K=0,1, \ldots$ consider a threeparameter family of finitely supported probability measures $\sum_{j=0}^{K} \pi_{j, K}(a, b) \delta_{j}$ on $\{0,1, \ldots, K\}$ with probabilities

$$
\begin{aligned}
\pi_{j, K}(a, b) & =\frac{(a+1-b)_{K}}{(a+1)_{K}} \frac{(-1)^{j}(-K)_{j}(a)_{j}(b)_{j}(1+a / 2)_{j}}{j !(a+1-b)_{j}(a / 2)_{j}(a+1+K)_{j}} \\
& =\left(\begin{array}{c}
K \\
j
\end{array}\right) \frac{(a-b+j+1)_{K-j}}{(a+2 j+1)_{K-j}} \frac{(b)_{j}}{(a+j)_{j}} .
\end{aligned}
$$

The natural ranges for the parameters are $a>-1,0<b<a+1, K \in \mathbf{N}$, but we also allow $K=0$ with a degenerate law $\delta_{0}$. The fact that these numbers add up to 1 can be deduced, e.g., from [1, identity (9.s)] by taking the limit as $c \rightarrow \infty, e=e(c) \rightarrow-\infty$ and $d \rightarrow \infty$. However, it is convenient to observe that

$$
\pi_{j, K}(a, b)=\lim _{c \rightarrow-\infty} p_{j, K}\left(\frac{a}{2}, b-\frac{a}{2}, c\right) .
$$


We will rely on this relation for quick proofs of the identities we need.

For each value of $K$, there is a Markov process $\xi_{t}^{(K)}$ based on these probabilities: the process starts with $\xi_{-A-B}^{(K)}=0$ and has transition probabilities

$$
\mathbf{P}\left(\xi_{t}^{(K)}=j \mid \xi_{s}^{(K)}=m\right)=\pi_{j-m, K-m}(2 A+2 m+t, t-s) .
$$

(It is straightforward to check that these number are nonnegative, and that the univariate laws are $\mathbf{P}\left(\xi_{t}^{(K)}=j\right)=\pi_{j, K}(2 A+t, t+A+B)$.) The ChapmanKolmogorov equations follow by taking a formal limit $C \rightarrow-\infty$ in (2.7).

Further, we define the Markov process of our interest and state the relevant moment formulas.

Proposition 3.1. For $t \in \mathscr{T}=(-A-B, \infty)$, define

$$
Y_{t}^{(K)}=\left(A+t+\xi_{t}^{(K)}\right)\left(A+\xi_{t}^{(K)}\right) .
$$

Then

(i) $\left(Y_{t}^{(K)}\right)_{t \in \mathscr{T}}$ is a Markov process;

(ii) for $t>-A-B$,

$$
\mathbf{E}\left(Y_{t}^{(K)}\right)=A^{2}+(A+K) t+K(A+B)
$$

(iii) for $-A-B<s<t$,

$$
\operatorname{Cov}\left(Y_{s}^{(K)}, Y_{t}^{(K)}\right)=K(K+A-B)(s+A+B) .
$$

The simplest way to prove Proposition 3.1 is to rely on limiting relation (3.2); this corresponds to replacing $N$ by $K$ and taking the limits as $C \rightarrow-\infty$ in Proposition 2.2.

From the mean and the variance formulas (3.5) and (3.6) we see that $Y_{t}^{(K)} / t \rightarrow A+K$ as $t \rightarrow \infty$. In particular, for any random variable $\Theta$ with finite support, we get

$$
\frac{Y_{t}^{(\Theta)}}{t} \rightarrow A+\Theta
$$

in mean square as $t \rightarrow \infty$.

Further, we confirm that Markov chain $\left(\zeta_{t}\right)$ from Section 2.2. can be represented as Markov chain $\left(\xi_{t}^{(K)}\right)$ with random $K$.

Denote

$$
\Pi_{k}(a, c ; N)=\frac{(c)_{N}(a)_{k}(-N)_{k}}{(c-a)_{N} k !(c)_{k}} .
$$

These numbers are probabilities if $c>0, a<1-N, k=0, \ldots, N$, see $[1$, (1.s)]. For $k \geqslant 1$,

$$
k \Pi_{k}(a, c ; N)=\frac{a N}{a-c-N+1} \Pi_{k-1}(a+1, c+1 ; N-1),
$$


so if $\mathbf{P}(U=k)=\Pi_{k}(a, c ; N)$, then

$$
\mathbf{E}(U)=\frac{a N}{a-c-N+1}, \quad \operatorname{Var}(U)=\frac{a(a-c+1)(c+N-1) N}{(c+N-a-2)(c+N-a-1)^{2}} .
$$

Consider an auxiliary random variable $\Theta$ with values in $\{0,1, \ldots, N\}$ such that

$$
\mathbf{P}(\Theta=k)=\Pi_{k}(A+C, A-B+1 ; N), \quad k=0, \ldots, N .
$$

(We calculated this law from $\Theta=\lim _{t \rightarrow \infty} \zeta_{t}$, but an analytic proof does not need to rely on this.)

Proposition 3.2. If $\Theta$ is random with law (3.10), and conditionally on $\Theta=K$, process $\left(\xi_{t}^{(\Theta)}\right)$ is a Markov chain with transitions (3.3), then the unconditional joint laws are: for $0 \leqslant j_{1} \leqslant \cdots \leqslant j_{n} \leqslant N$,

$$
\sum_{k=j_{n}}^{N} \mathbf{P}(\Theta=k) \mathbf{P}\left(\xi_{t_{1}}^{(k)}=j_{1}, \ldots, \xi_{t_{n}}^{(k)}=j_{n}\right)=\mathbf{P}\left(\zeta_{t_{1}}=j_{1}, \ldots, \zeta_{t_{n}}=j_{n}\right)
$$

where $\left(\zeta_{t}\right)$ is the Markov process from Section 2 with parameters $N, A, B, C$.

$\mathrm{P}$ r o o f. Let $\zeta_{t}=\xi_{t}^{(\Theta)}$. Then $\left(\zeta_{t}\right)_{t \in \mathscr{T}}$ is a Markov chain regardless of the law of the randomization $\Theta$. This follows from the fact that in reverse time the transition probabilities $\xi_{t}^{(K)} \mid \xi_{u}^{(K)}$ do not depend on $K$. To see that joint laws match, we observe that both Markov processes $\left(\zeta_{t}\right)$ and $\left(\xi_{t}^{(\Theta)}\right)$ have the same limiting distribution (3.10) as $t \rightarrow \infty$ and the same reverse transition probabilities, as the reverse transition probabilities $(2.10)$ for $\left(\zeta_{t}\right)$ do not depend on parameter $C$, and the transition probabilities for Markov chain $\left(\xi_{t}^{(\Theta)}\right)$ are the limit as $C \rightarrow-\infty$ of (2.10). Proposition 3.2 is proved.

3.2. The second conditional process. For $K=0, \ldots, N$, we define the dual Markov chain $\left(\widetilde{\xi}_{t}^{(K)}\right)_{t \in \widetilde{T}}$ with state space $\{K, \ldots, N\}$ and time $\widetilde{\mathscr{T}}=(A+N-C, \infty)$. This Markov chain starts at $\widetilde{\xi}_{A+N-C}^{(K)}=N$ and jumps down according to the transition matrix $\widetilde{P}_{s, t}=\left[\widetilde{p}_{s, t}(i, j)\right]$ with entries

$$
\widetilde{p}_{s, t}(i, j)=\pi_{j-K, i-K}(2 A+2 K-t, 2 A+i+K-s),
$$

$i=K, K+1, \ldots, N, j=K, K+1, \ldots, i$. (The remaining entries of this $(N-K+1) \times(N-K+1)-$ matrix are zero.) As in Section 2.2., these are just the reverse transitions for Markov chain $\left(\xi_{-t}^{(K)}\right)$. In particular, the univariate laws of the dual Markov chain are $\mathbf{P}\left(\widetilde{\xi}_{t}^{(K)}=j\right)=\pi_{j-K, N-K}(2 A+$ $2 K-t, A+C+K)$.

Further, noting again that the lines $\ell_{j}(t)=(t-A-j)(A+j)$ do not intersect over $\widetilde{\mathscr{T}}$, we define the corresponding Markov process

$$
\widetilde{Y}_{t}^{(K)}=\left(t-A-\widetilde{\xi}_{t}^{(K)}\right)\left(A+\widetilde{\xi}_{t}^{(K)}\right),
$$


which will give the «second half» of the quadratic harness of Theorem 1.1.

One can repeat analytic argument, however, it is simpler to use the relation $\widetilde{Y}_{t}^{(K)}=-Y_{-t}^{(K)}$, which in particular gives

$$
\mathbf{E}\left(\widetilde{Y}_{t}^{(K)}\right)=(A+K) t-(A+K)(A+N)-C(N-K) .
$$

Proposition 3.3. If $\Theta$ has law (3.10), then $\left(\widetilde{Y}_{t}^{(\Theta)}\right)_{t \in \widetilde{T}}$ is Markov and has the law $\left(Y_{t}^{\prime}\right)_{t \in \widetilde{\mathscr{T}}}$.

In particular, Proposition 2.5 implies that $\left(\widetilde{Y}_{t}^{(\Theta)}\right)_{t \in \widetilde{T}}$ can be transformed into quadratic harness on $(1, \infty)$ with parameters as in Theorem 1.1.

P r o o f. In view of $(2.25)$, we only need to verify that $\left(\widetilde{\xi}_{t}^{(\Theta)}\right)_{t \in \widetilde{T}}$ has the same finite-dimensional laws as process $\left(\zeta_{t}^{\prime}\right)_{t \in \widetilde{\mathscr{T}}}$. A noncomputational justification is to note that algebraic relations between the probabilities in Proposition 3.2 hold true also for $s<t<C-A-N$. Proposition 3.3 is proved.

3.3. Extending quadratic harness: stitching two processes together. We now define the joint law of processes $\left(Y_{t}\right)$ and $\left(Y_{t}^{\prime}\right)$ by requesting that conditionally on $\Theta=K$, processes $\left(Y_{t}^{(K)}\right)_{t \in \mathscr{T}}$ and $\left(Y_{t}^{(K)}\right)_{t \in \widetilde{T}}$ are independent. Taking $\Theta$ with distribution (3.10), this defines a joint distribution for a pair of processes $\left(Y_{t}^{(\Theta)}\right)_{t \in \mathscr{T}}$ and $\left(\widetilde{Y}_{t}^{(\Theta)}\right)_{t \in \widetilde{T}}$ with marginals distributed as $\left(Y_{t}\right)$ and $\left(Y_{t}^{\prime}\right)$, respectively.

Consider the centered versions $X_{t}=Y_{t}^{(\Theta)}-\mathbf{E}\left(Y_{t}^{(\Theta)}\right)$ and $X_{t}^{\prime}=\tilde{Y}_{t}^{(\Theta)}$ $\mathbf{E}\left(\widetilde{Y}_{t}^{(\Theta)}\right)$ of the two processes.

Further, we recall the transformations we will use to stich the processes together. We write $(2.24)$ as

$$
\ell(t)=\frac{t(A+N-C)-A-B}{v} \text { and } m(t)=\frac{1-t}{v}
$$

for $0<t<1$. Similarly, we write $(2.29)$ as

$$
\ell^{\prime}(t)=\frac{t(A+N-C)-A-B}{v} \quad \text { and } \quad m^{\prime}(t)=\frac{t-1}{v}
$$

for $t>1$. Here

$$
v=\frac{\sqrt{N(N+A-B)(A+C)(B+C)}}{\sqrt{N-1-B-C}}
$$

is a normalizing factor, compare (2.22). The corresponding Möbius transformations are

$$
\varphi(t):=\frac{\ell(t)}{m(t)}=\frac{t(A-C+N)-A-B}{1-t} \quad \text { and } \quad \varphi^{\prime}(t):=\frac{\ell^{\prime}(t)}{m^{\prime}(t)}=-\varphi(t) .
$$


The stitched process is then given by

$$
Z_{t}= \begin{cases}m(t) X_{\varphi(t)}, & 0<t<1 \\ Z / v, & t=1 \\ m^{\prime}(t) X_{\varphi^{\prime}(t)}^{\prime}, & t>1\end{cases}
$$

where $Z=(A+C) N+\Theta(N-B-C)$.

It is convenient to observe that $\left(Z_{t}\right)_{t>0}$ is a Markov process. This follows from Markov property and $\Theta$-conditional independence of $\left(Y_{t}^{(\Theta)}\right)_{t \in \mathscr{T}}$ and $\left(\widetilde{Y}_{t}^{(\Theta)}\right)_{t \in \tilde{T}}$.

3.4. Proof of Theorem 1.1. Our goal is to verify that process (3.18) satisfies assumptions of the following lemma.

Lemma 3.1 (see [5]). Suppose a square-integrable Markov harness $\mathbf{Z}=$ $\left(Z_{t}\right)_{t>0}$ is a quadratic harness on $(0,1)$ and on $(1, \infty)$, with the same parameters $\eta, \theta, \sigma, \tau, \gamma$. If $\operatorname{Var}\left(Z_{1} \mid Z_{s}, Z_{u}\right)$ is given by the formula (1.3) with $t=1$ and with the same parameters $\eta, \theta, \sigma, \tau, \gamma$, then $\mathbf{Z}$ is a quadratic harness on $(0, \infty)$.

We will verify each assumption separately.

3.4.1. Auxiliary moment calculations. We first check that $\mathbf{E}(Z)=0$, $\operatorname{Var}(Z)=v^{2}$ so $\operatorname{Var}\left(Z_{1}\right)=1$. This is a consequence of the following lemma.

Lemma 3.2. For $\Theta$ with law (3.10),

$$
\mathbf{E}(\Theta)=\frac{(A+C) N}{B+C-N}, \quad \operatorname{Var}(\Theta)=M^{2},
$$

where $M$ is given by $(2.22)$.

P r o o f. See (3.9).

Lemma 3.3. For $\Theta$ with law (3.10), if $0 \leqslant m \leqslant k \leqslant n \leqslant N$, and $s \in \mathscr{T}$, $u \in \widetilde{\mathscr{T}}$, then using notation $(3.8), \mathbf{P}\left(\Theta=k \mid \zeta_{s}, \zeta_{u}^{\prime}\right)=\Pi_{k-\zeta_{s}}\left(2 A+\zeta_{s}+\zeta_{u}^{\prime}-\right.$ $\left.u, 2 A+2 \zeta_{s}+s+1 ; \zeta_{u}^{\prime}-\zeta_{s}\right)$.

$\mathrm{P}$ r o o f. The proof consists of careful isolation of factors that depend only on $k$ in the joint distribution

$$
\begin{aligned}
\mathbf{P}(\Theta & \left.=k, \zeta_{s}=m, \zeta_{u}^{\prime}=n\right) \\
= & \Pi_{k}(A+C, A-B+1 ; N) \pi_{m, k}(2 A+s, s+A+B) \\
& \times \pi_{n-k, N-k}(2 A+2 k-u, A+C+k) \\
= & \text { const }_{N, m, n} \frac{(2 A+n+m-u)_{k-m}(-(n-m))_{k-m}}{(k-m) !(2 A+2 m+s+1)_{k-m}} .
\end{aligned}
$$

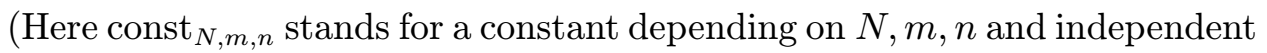
of $k$.) Details are omitted. Lemma 3.3 is proved.

We will need the first two conditional moments. 
Corollary 3.1. For $\Theta$ with law (3.10),

$$
\mathbf{E}\left(\Theta \mid Y_{s}, Y_{u}^{\prime}\right)=\frac{Y_{s}+Y_{u}^{\prime}}{u+s}-A,
$$

and

$$
\operatorname{Var}\left(\Theta \mid Y_{s}, Y_{u}^{\prime}\right)=\frac{s^{2} Y_{u}^{\prime}-u^{2} Y_{s}+s u\left(Y_{u}^{\prime}-Y_{s}\right)+\left(Y_{s}+Y_{u}^{\prime}\right)^{2}}{(s+u-1)(s+u)^{2}}
$$

P r o o f. For fixed $\zeta_{s}=m, \zeta_{u}^{\prime}=n$, Lemma 3.3 gives $\mathbf{E}\left(\Theta \mid \zeta_{s}=m\right.$, $\left.\zeta_{u}^{\prime}=n\right)=m+\mathbf{E}(U)$, where $\mathbf{P}(U=k)=\Pi_{k}(2 A+m+n-u, 2 A+2 m+$ $s+1 ; n-m)$, so from $(3.9)$ we get

$$
\begin{aligned}
\mathbf{E}\left(\Theta \mid \zeta_{s}, \zeta_{u}^{\prime}\right) & =\zeta_{s}-\frac{\left(2 A+\zeta_{s}+\zeta_{u}^{\prime}-u\right)\left(\zeta_{u}^{\prime}-\zeta_{s}\right)}{s+u} \\
& =\frac{1}{s+u}\left(\zeta_{s}\left(2 A+s+\zeta_{s}\right)+\zeta_{u}^{\prime}\left(u-2 A-\zeta_{u}^{\prime}\right)\right) .
\end{aligned}
$$

This gives (3.19).

Using (3.9) again, we get

$$
\begin{aligned}
& \operatorname{Var}\left(\Theta \mid \zeta_{s}, \zeta_{u}^{\prime}\right) \\
& \quad=\frac{\left(\zeta_{s}-\zeta_{u}^{\prime}\right)\left(\zeta_{s}+\zeta_{u}^{\prime}+2 A+s\right)\left(\zeta_{s}+\zeta_{u}^{\prime}+2 A-u\right)\left(s+u-\zeta_{u}^{\prime}+\zeta_{s}\right)}{(s+u)^{2}(s+u-1)} .
\end{aligned}
$$

This gives (3.20). Corollary 3.1 is proved.

3.4.2. Verification of assumptions in Lemma 3.1. We first compute the covariance.

Lemma 3.4. The stitched process (3.18) has covariance (1.1).

P r o o f. From the transformations (2.23) and (2.30) exhibited in the proofs of Proposition 2.3 and Proposition 2.5, we see that the covariance is as required for $0 \leqslant s<u<1$ and for $1<s<u$, so by time-reversibility argument it remains only to consider the case $s \leqslant 1<u$.

Since $\lim _{t \rightarrow \infty} Y_{t} / t=A+\Theta$, see (3.7), we get $Z_{1}=\lim _{s \rightarrow 1-} Z_{s}$ in mean square. Therefore, we only need to consider the covariance for $s<1<u$. Denote

$$
s^{\prime}=\varphi(s)=\frac{\ell(s)}{m(s)}, \quad u^{\prime}=\varphi^{\prime}(u)=\frac{\ell^{\prime}(u)}{m^{\prime}(u)} .
$$

From (3.18) we get $\operatorname{Cov}\left(Z_{s}, Z_{u}\right)=m(s) m^{\prime}(u) \operatorname{Cov}\left(Y_{s^{\prime}}, Y_{u^{\prime}}^{\prime}\right)$. By conditional independence, from (2.20) (used with $X=Y_{s^{\prime}}, Y=Y_{u^{\prime}}^{\prime}, U=\Theta$ ) we have $\operatorname{Cov}\left(Y_{s^{\prime}}, Y_{u^{\prime}}^{\prime}\right)=\operatorname{Cov}\left(\mathbf{E}\left(Y_{s^{\prime}} \mid \Theta\right), \mathbf{E}\left(Y_{u^{\prime}}^{\prime} \mid \Theta\right)\right)$. So from (3.5) and (3.14) we get $\operatorname{Cov}\left(Y_{s^{\prime}}, Y_{u^{\prime}}^{\prime}\right)=\left(u^{\prime}-A-N+C\right)\left(s^{\prime}+A+B\right) \operatorname{Var}(\Theta)$. By Lemma 3.2

$$
\begin{aligned}
\operatorname{Cov}\left(Z_{s}, Z_{u}\right) & =M^{2} m(s) m^{\prime}(u)\left(u^{\prime}-A-N+C\right)\left(s^{\prime}+A+B\right) \\
& =M^{2} m(s) m^{\prime}(u)\left(\varphi^{\prime}(u)-A-N+C\right)(\varphi(s)+A+B) \\
& =M^{2}\left(\ell^{\prime}(u)-(A+N-C) m^{\prime}(u)\right)(\ell(s)+(A+B) m(s)) .
\end{aligned}
$$


Now we notice that (2.24), see also (3.15), gives $\ell(s)+(A+B) m(s)=s / M$, and similarly (2.29), see also (3.16), gives $\ell^{\prime}(u)-(A+N-C) m^{\prime}(u)=1 / M$. Therefore, $\operatorname{Cov}\left(Z_{s}, Z_{u}\right)=s$ and (1.1) holds. Lemma 3.4 is proved.

Further, we verify harness property on the entire interval $(0, \infty)$.

Lemma 3.5. Suppose that the law of $\Theta$ is (3.10). Then (3.18) defines a harness on $(0, \infty)$.

$\mathrm{P}$ r o o f. The transformations (2.23) and (2.30) used in the proofs of Proposition 2.3 and Proposition 3.3, show that (1.2) holds for $s<t<u<1$ and for $1<s<t<u$.

To end the proof, we only need to verify (1.2) for $s<t=1<u$. Indeed, if we have this case, then the case $0<s<t<1<u$, is handled from Markov property as

$\mathbf{E}\left(Z_{t} \mid Z_{s}, Z_{u}\right)=\mathbf{E}\left(\mathbf{E}\left(Z_{t} \mid Z_{s}, Z_{1}\right) \mid Z_{s}, Z_{u}\right)=\frac{1-t}{1-s} Z_{s}+\frac{t-s}{1-s} \mathbf{E}\left(Z_{1} \mid Z_{s}, Z_{u}\right)$.

The other case $0<s<1<t<u$ is handled similarly (or by time inversion). Finally, the cases $1=s<t<u$ and $s<t<u=1$ are the limits of cases $0<s<1<t<u$ and $0<s<t<1<u$, respectively.

To prove (1.2) for $s<t=1<u$, we use notation (3.21). The joint distribution $Z_{s}, Z_{1}, Z_{u}$ is determined from the joint distribution of $Y_{s^{\prime}}, \Theta, Y_{u^{\prime}}^{\prime}$. To verify harness property, we notice that Corollary $3.1 \mathrm{im}-$ plies that $\mathbf{E}\left(Z_{1} \mid Y_{s^{\prime}}, Y_{u^{\prime}}^{\prime}\right)$ is a linear function of $Y_{s^{\prime}}, Y_{u^{\prime}}^{\prime}$, so it is also a linear function of $Z_{s}, Z_{u}$. Since by Lemma 3.4 the covariance of $\left(Z_{t}\right)$ is (1.1), this determines the coefficients of the linear regression, and (1.2) follows. Lemma 3.5 is proved.

Finally, we verify that the conditional variance of $Z_{1}$ satisfies the assumption of Lemma 3.1.

Lemma 3.6. If $\Theta$ has law (3.10), then (1.3) holds for $t=1$.

$\mathrm{P}$ r o of. Fix $0<s<1<u$. Using notation (3.21), we see that $\operatorname{Var}\left(Z_{1} \mid Z_{s}, Z_{u}\right)=\operatorname{Var}\left(Z_{1} \mid Y_{s^{\prime}}, Y_{u^{\prime}}^{\prime}\right)$ is a constant multiple of the right-hand side of (3.20) (with $s, u$ exchanged to $s^{\prime}, u^{\prime}$ ). We do not have to pay attention to the deterministic multiplicative constant, say const ${ }_{s, u}$, which is determined uniquely from the covariance of $\left(Z_{t}\right)$. So we write

$$
\operatorname{Var}\left(Z_{1} \mid Z_{s}, Z_{u}\right)=\text { const }_{s, u}\left(s^{\prime 2} Y_{u^{\prime}}^{\prime}-u^{\prime 2} Y_{s^{\prime}}+s^{\prime} u^{\prime}\left(Y_{u^{\prime}}^{\prime}-Y_{s^{\prime}}\right)+\left(Y_{s^{\prime}}+Y_{u^{\prime}}^{\prime}\right)^{2}\right) \text {. }
$$

Further, we use the inverse of the transformation (3.18), see (2.13), (2.26) and (3.21),

$$
\begin{aligned}
Y_{s^{\prime}} & =\frac{Z_{s}}{m(s)}+\frac{A(B+C)(A+N)+N B C}{B+C-N}+\frac{\ell(s)}{m(s)} \frac{A(B+C)+N C}{B+C-N} \\
& =\frac{v Z_{s}-(A B+C(A+N) s)}{1-s},
\end{aligned}
$$




$$
\begin{aligned}
Y_{u^{\prime}}^{\prime} & =\frac{Z_{u}}{m^{\prime}(u)}+\frac{\ell^{\prime}(u)}{m^{\prime}(u)} \frac{A(B+C)+N C}{B+C-N}-\frac{A(B+C)(A+N)+N B C}{B+C-N} \\
& =\frac{v Z_{u}-(A B+C(A+N) u)}{u-1} .
\end{aligned}
$$

Using these expressions, we rewrite the right-hand side of (3.22) as a deterministic multiple of

$$
\begin{aligned}
1+ & \frac{v^{2}}{(A+C)(B+C) N(A-B+N)}\left(\frac{\left(Z_{u}-Z_{s}\right)^{2}}{(u-s)^{2}}+\frac{\left(u Z_{s}-s Z_{u}\right)^{2}}{(u-s)^{2}}\right) \\
& -\frac{((A-B)(B+C)+(A+B+2 C) N) v}{(A+C)(B+C) N(A-B+N)} \frac{Z_{u}-Z_{s}}{u-s} \\
& -\frac{\left(N^{2}+(A-B) N+(A+C)(B+C)\right) v}{(A+C)(B+C) N(A-B+N)} \frac{u Z_{s}-s Z_{u}}{u-s} \\
& +\frac{2 v^{2}}{(A+C)(B+C) N(A-B+N)} \frac{\left(Z_{u}-Z_{s}\right)\left(u Z_{s}-s Z_{u}\right)}{(u-s)^{2}} .
\end{aligned}
$$

From (3.17), we see that up to a deterministic factor this quadratic form matches (1.3) with parameters (1.9)-(1.12). Lemma 3.6 is proved.

3.5. Conclusion of the proof of Theorem 1.1. By Lemma 3.4, the covariance of our process is (1.1). From Lemma 3.5 we see that (1.2) holds for all $0<s<t<u$. Furthermore, from the transformations (2.23) and (2.30) exhibited in the proofs of Proposition 2.3 and Proposition 3.3, we see that $\left(Z_{t}\right)$ is a quadratic harness on $(0,1)$ and on $(1, \infty)$ with the same parameters. Since (1.2) holds for all $0<s<t<u$, from Lemmas 3.6 and 3.1 we see that (1.3) also holds for all $0<s<t<u$. This ends the proof. (The one-sided conditional moments do not need to be verified for processes on $(0, \infty)$ as they fall in place by $[2,(2.7),(2.8),(2.27)$, and $(2.28)]$.

Acknowledgement. The topic of this research was initiated by a conversation with Albert Cohen. We would like to thank J. Wesołowski for information about [6] and several related discussions. We also thank J. A. Wilson for a discussion on the role of discrete models.

\section{Appendix A. Auxiliary identities.}

A.1. Properties of Wilson's distributions from Section 2.1.

Lemma A.1. For $k=0,1, \ldots, N, j=0,1, \ldots, k$, we have

$$
\frac{p_{j, k}(a, b, a+k+2 \delta) p_{k, N}(a+\delta, b+\delta, c)}{p_{j, N}(a, b, c+\delta)}=p_{k-j, N-j}(a+j+\delta,-a-j+\delta, c) .
$$

In particular, by taking the sum over $j$ in (A.1) we have

$$
p_{k, N}(a+\delta, b+\delta, c)=\sum_{j=0}^{k} p_{j, N}(a, b, c+\delta) p_{k-j, N-j}(a+j+\delta,-a-j+\delta, c) .
$$


P r o of of L e m ma A.1. Multiplying both sides of (A.1) by $p_{j, N}(a, b, c+\delta)$, expanding them by the use of (2.1) and (2.2), canceling out common terms and grouping the remaining ones, we observe that (A.1) would follow if we verify that LHS $=$ RHS with

$$
\begin{aligned}
\text { LHS }= & \frac{1}{(2 a+1)_{k}(2 a+k+1)_{j}} \frac{(2 a+2 \delta)_{k}(2 a+2 \delta+k)_{j}}{(2 a+2 \delta+1)_{N}(2 a+2 \delta+N+1)_{k}} \\
& \times(a+c+\delta)_{k} \frac{(a+\delta+1)_{k}}{(a+\delta)_{k}} \frac{(-2 \delta-k+1)_{k}}{(-2 \delta-k+1)_{j}} \frac{(a-c+\delta+1)_{N}}{(a-c+\delta+1)_{k}} \\
& \times \frac{(a+b+2 \delta)_{k}}{(-a-b-2 \delta-k+1)_{k}} \frac{(-k)_{j}(-N)_{k}}{k !}
\end{aligned}
$$

and

$$
\begin{aligned}
\mathrm{RHS}= & \frac{(2 a+2 j+1)_{N-j}}{(2 a+1)_{N}(2 a+N+1)_{j}(2 a+2 j+1)_{k-j}} \\
& \times \frac{(2 a+2 \delta+2 j)_{k-j}}{(2 a+2 \delta+2 j+1)_{N-j}(2 a+2 \delta+j+N+1)_{k-j}} \\
& \times(a+c+\delta)_{j}(a+c+\delta+j)_{k-j} \\
& \times \frac{(a+\delta+j+1)_{k-j}}{(a+\delta+j)_{k-j}}(2 \delta)_{k-j} \frac{(a-c+\delta+j+1)_{N-j}}{(a-c+\delta+j+1)_{k-j}} \\
& \times \frac{(a-c-\delta+1)_{N}}{(a-c-\delta+1)_{j}(a-c-\delta+j+1)_{N-j}} \frac{(j-N)_{k-j}(-N)_{j}}{(k-j) !} .
\end{aligned}
$$

To perform the verification, we will use the following simplification rules

$$
\begin{aligned}
(\alpha)_{N}(\alpha+N)_{M} & =(\alpha)_{M+N}, \\
\frac{(\alpha+L+1)_{M-L}}{(\alpha+L)_{M-L}} & =\frac{\alpha+M}{\alpha+L}, \\
\frac{(\alpha)_{L}}{(\alpha+1)_{M+L}} & =\frac{\alpha}{(\alpha+L)_{M+1}} .
\end{aligned}
$$

From (A.3) it follows that

$$
\begin{gathered}
\frac{(2 a+2 j+1)_{N-j}}{(2 a+1)_{N}(2 a+N+1)_{j}(2 a+2 j+1)_{k-j}}=\frac{(2 a+k+j+1)_{N-k}}{(2 a+1)_{N+j}} \\
=\frac{(2 a+k+j+1)_{N-k}}{(2 a+1)_{k+j}(2 a+1+k+j)_{N-k}}=\frac{1}{(2 a+1)_{k}(2 a+k+1)_{j}} .
\end{gathered}
$$

Similarly, (A.3) and (A.5) give

$$
\begin{aligned}
& \frac{(2 a+2 \delta+2 j)_{k-j}}{(2 a+2 \delta+2 j+1)_{N-j}(2 a+2 \delta+j+N+1)_{k-j}} \\
& =\frac{(2 a+2 \delta+2 j)_{k-j}}{(2 a+2 \delta+2 j+1)_{N+k-2 j}}=\frac{2 a+2 \delta+2 j}{(2 a+2 \delta+k+j)_{N+1-j}},
\end{aligned}
$$


while applied to the analogous expression in LHS they give

$$
\begin{aligned}
\frac{(2 a+2 \delta)_{k}(2 a+2 \delta+k)_{j}}{(2 a+2 \delta+1)_{N}(2 a+2 \delta+N+1)_{k}} & =\frac{(2 a+2 \delta)_{k+j}}{(2 a+2 \delta+1)_{k+N}} \\
& =\frac{2 a+2 \delta}{(2 a+2 \delta+k+j)_{N+1-j}} .
\end{aligned}
$$

From (A.3) we get

$$
\begin{gathered}
(a+c+\delta)_{j}(a+c+\delta+j)_{k-j}=(a+c+\delta)_{k}, \\
\frac{(a-c+\delta+j+1)_{N-j}}{(a-c+\delta+j+1)_{k-j}}=(a-c+\delta+k+1)_{N-k}=\frac{(a-c+\delta+1)_{N}}{(a-c+\delta+1)_{k}}
\end{gathered}
$$

and (this expression appears only in RHS)

$$
\frac{(a-c-\delta)_{N}}{(a-c-\delta+1)_{j}(a-c-\delta+j+1)_{N-j}}=1 .
$$

From (A.4) it follows that

$$
\frac{(a+\delta+j+1)_{k-j}}{(a+\delta+j)_{k-j}}=\frac{a+\delta+k}{a+\delta+j} \quad \text { and } \quad \frac{(a+\delta+1)_{k}}{(a+\delta)_{k}}=\frac{a+\delta+k}{a+\delta} .
$$

For the remaining expressions from LHS we have

$$
\frac{(-2 \delta-k+1)_{k}}{(-2 \delta-k+1)_{j}}=(-1)^{k+j}(2 \delta)_{k-j} \quad \text { and } \quad \frac{(a+b+2 \delta)_{k}}{(-a-b-2 \delta-k+1)_{k}}=(-1)^{k} \text {. }
$$

Now, the above simplifications show that equality LHS $=$ RHS is equivalent to

$$
\begin{gathered}
\frac{(-k)_{j}(-N)_{k}}{k !}(-1)^{k+j}(2 \delta)_{k-j} \frac{2 a+2 \delta}{(2 a+2 \delta+k+j)_{N+1-j}} \frac{a+\delta+k}{a+\delta}(-1)^{k} \\
=\frac{(j-N)_{k-j}(-N)_{j}}{(k-j) !}(2 \delta)_{k-j} \frac{2 a+2 \delta+2 j}{(2 a+2 \delta+k+j)_{N+1-j}} \frac{a+\delta+k}{a+\delta+j},
\end{gathered}
$$

which is easily seen to be true. Thus (A.1) is proved. Lemma A.1 is proved.

Further, we compute the moments of an auxiliary random variable associated with probability law (2.1).

Lemma A.2. Suppose parameters $a, b, c, N$ satisfy (2.3). Consider a random variable $Y$ such that

$$
\mathbf{P}(Y=k(2 a+k))=p_{k, N}(a, b, c), \quad \text { where } k=0, \ldots, N .
$$

Then

$$
\mathbf{E}(Y)=\frac{(a+b)(a+c) N}{b+c-N}
$$

and

$$
\operatorname{Var}(Y)=\frac{N(a-b+N)(a+b)(a-c+N)(a+c)(b+c)}{(b+c-N)^{2}(N-b-c-1)} .
$$


P r o of of $\mathrm{L}$ e $\mathrm{mm}$ a A.2. The proof is elementary for $N=0,1$, as the law of $Y$ is $\delta_{0}$ for $N=0$ and

$$
\frac{(a-b+1)(a-c+1)}{(2 a+1)(-b-c+1)} \delta_{0}+\frac{(a+b)(a+c)}{(2 a+1)(b+c-1)} \delta_{2 a+1} \quad \text { for } N=1 .
$$

For $N>1$ and $k \geqslant 1$, we have

$$
k(2 a+k) p_{k, N}(a, b, c)=\frac{(a+b)(a+c) N}{b+c-N} p_{k-1, N-1}(a+1, b, c),
$$

which gives (A.7). For $k>2$, iterating the algebraic identity we get

$$
\begin{aligned}
& k(k-1)(2 a+k)(2 a+k+1) p_{k, N}(a, b, c) \\
& \quad=\frac{(a+b)(a+b+1)(a+c)(a+c+1)(N-1) N}{(b+c-N)(b+c-N+1)} p_{k-2, N-2}(a+2, b, c) .
\end{aligned}
$$

Noting that $k(k-1)(2 a+k)(2 a+k+1)=k^{2}(2 a+k)^{2}-(1+2 a) k(2 a+k)$, we evaluate

$$
\mathbf{E}\left(Y^{2}\right)=\frac{(a+b)(a+b+1)(a+c)(a+c+1)(N-1) N}{(b+c-N)(b+c-N+1)}+(1+2 a) \mathbf{E}(Y)
$$

and (A.8) follows by an elementary calculation. Lemma A.2 is proved.

\section{Appendix B. Transforming a process into a quadratic harness.}

In this section we recall a procedure that transforms (some) Markov processes with linear regressions and quadratic conditional variances into the quadratic harnesses. The following is [4, Theorem 3.1] specialized to $\chi=0$, $\eta=\eta_{0}, \theta=\theta_{0}, \sigma=0, \tau=1, \rho=0, a=M, b=M \psi, c=M, d=M \delta$.

Proposition B.1. Suppose $\left(Y_{t}\right)$ is a (real-valued) Markov process on an open interval $\mathscr{T} \subset \mathbf{R}$ such that

(i) $\mathbf{E}\left(Y_{t}\right)=\alpha+\beta t$ for some real $\alpha, \beta$;

(ii) for $s<t$ in $\mathscr{T}, \operatorname{Cov}\left(Y_{s}, Y_{t}\right)=M^{2}(\psi+s)(\delta+t)$, where $M^{2}(\psi+t)(\delta+$ $t)>0$ on the entire interval $\mathscr{T}$, and that $\delta-\psi>0$;

(iii) (1.2) holds for $s<t<u$;

(iv) for $s<t<u$,

$$
\operatorname{Var}\left(Y_{t} \mid Y_{s}, Y_{u}\right)=F_{t, s, u}\left(\eta_{0} \frac{u Y_{s}-s Y_{u}}{u-s}+\theta_{0} \frac{Y_{u}-Y_{s}}{u-s}+\frac{\left(Y_{u}-Y_{s}\right)^{2}}{(u-s)^{2}}\right)
$$

where $F_{t, s, u}$ is nonrandom and $\theta_{0}, \eta_{0} \in \mathbf{R}$ are such that $\chi:=\alpha \eta_{0}+\beta \theta_{0}$ $+\beta^{2}>0$.

Denote $X_{t}=Y_{t}-\mathbf{E}\left(Y_{t}\right)$. Then there are two affine functions

$$
\ell(t)=\frac{t \delta-\psi}{M(\delta-\psi)} \quad \text { and } \quad m(t)=\frac{1-t}{M(\delta-\psi)},
$$


and an open interval $\mathscr{T}^{\prime} \subset(0, \infty)$ such that $Z_{t}:=m(t) X_{\ell(t) / m(t)}$ defines a process $\left(Z_{t}\right)$ on $\mathscr{T}^{\prime}$ such that (1.1) and (1.2) hold and (1.3) holds with parameters $\eta=M\left(\delta \eta_{0}+\left(2 \beta+\theta_{0}\right)\right) / \chi, \theta=M\left(2 \beta+\psi \eta_{0}+\theta_{0}\right) / \chi, \sigma=M^{2} / \chi$, $\tau=\frac{M^{2}}{\chi}, \gamma=1+2 \sqrt{\sigma \tau}$.

$\mathrm{R}$ e $\mathrm{m}$ a r k B.1. The time domain $\mathscr{T}^{\prime}$ is the image of $\mathscr{T}$ under the Möbius transformation $t \mapsto(t+\psi) /(t+\delta)$.

\section{REFERENCES}

1. Askey R. Beta integrals and the associated orthogonal polynomials. - Lecture Notes in Math., 1989, v. 1395, pp. 84-121.

2. Bryc W., Matysiak W., Wesotowski J. Quadratic harnesses, q-commutations, and orthogonal martingale polynomials. - Trans. Amer. Math. Soc., 2007, v.359, № 11, p. 5449-5483.

3. Bryc W., Wesotowski J. Askey-Wilson polynomials, quadratic harnesses and martingales. - Ann. Probab., 2010, v. 38, № 3, p. 1221-1262.

4. Bryc W., Wesotowski J. Bridges of quadratic harnesses. — Electron. J. Probab., 2012, v. 17 , № 105 , p. 1-25.

5. Bryc W., Wesotowski J. Stitching pairs of Lévy processes into martingales. - Stochastic Process. Appl., 2012, v. 122, № 8, p. 2854-2869.

6. Jamiotkowska M. Bi-Pascal process - definition and properties. - Master's thesis, Warsaw University of Technology, 2009.

7. Koekoek R., Swarttouw R.F. The Askey scheme of hypergeometric orthogonal polynomials and its $q$-analogue. - Delft University of Technology Report no. 98-17, 1998; http://fa.its.tudelft.nl/ koekoek/askey.html.

8. Mansuy R., Yor M. Harnesses, Lévy bridges and Monsieur Jourdain. - Stochastic Process. Appl., 2005, v. 115, № 2, p. 329-338.

9. Szabtowski P. J. A few remarks on quadratic harnesses. - J. Difference Equ. Appl., 2014 , v. 20 , № 4 , p. 586-609.

10. Wesotowski J. Stochastic processes with linear conditional expectation and quadratic conditional variance. - Probab. Math. Statist., 1993, v. 14, № 1, p. 33-44.

11. Wilson J. A. Some hypergeometric orthogonal polynomials. - SIAM J. Math. Anal., 1980, v. 11, № 4, p. 690-701.

12. Zografi M., Xekalaki E. The generalized Waring process. - In Proceedings of the 5th Hellenic-European Conference on Computer Mathematics and its Applications, Athens, Greece, 2001, p. 886-893.

Поступила в редакцию

8.II.2012

Исправленный вариант

23.V.2014 